\title{
Formación de usuarios de la información mediante aplicaciones Web 2.0
}

\author{
Eder Ávila Barrientos \\ Coordinación del Posgrado en Bibliotecología y Estudios de la Información, Universidad Nacional \\ Autónoma de México - UNAM, México.
}

REVIEW

\section{Resumen}

Objetivo: Analizar las aplicaciones Web 2.0 para estimar su utilización en los programas de formación de usuarios de la información.

Método: Análisis documental y la hermenéutica del discurso aplicados a los documentos impresos y digitales que abordan la temática del objeto de estudio.

Resultados: Se responden una serie de cuestionamientos relacionados con la integración de las aplicaciones Web 2.0 en el diseño de programas de formación de usuarios de la información. En lo que respecta a la selección de aplicaciones Web 2.0, se empleo la técnica de análisis de atributos basada en las variables de uso didáctico.

Conclusiones: Las tecnologías de la Web 2.0 y su integración en los programas de formación de usuarios de la información, representan un nicho de oportunidad para los bibliotecólogos. Una oportunidad para alcanzar el futuro y romper viejos paradigmas de la formación de usuarios.

Palabras clave

Formación de usuarios ; Tecnologías de la información y comunicación ; Aplicaciones Web 2.0 ; Programas de formación de usuarios

\section{Training of users of information through Web 2.0 applications}

\begin{abstract}
Objective: To analyze web applications 2.0 to estimate its use in programs of training of users of the information.

Method: Documentary analysis and the hermeneutic of speech applied to print and digital documents that address the theme of the object of study.

Results: Answered a series of questions related to the integration of Web 2.0 applications in the design of training programmes for users of the information. In regards to the selection of web 2.0 applications, is job analysis technique based on instructional use variables.

Conclusions: The technologies of web 2.0 and its integration in the programs of training of users of information, represent a niche opportunity for the librarians. An opportunity to achieve the future and break old paradigms of the training of users.
\end{abstract}

Keywords

User training ; Information technology and communication ; Web 2.0 applications ; User training programs 


\section{Introducción}

El presente trabajo tiene como objetivo, analizar las aplicaciones web 2.0 para estimar su utilización en los programas de formación de usuarios de la información. Las tecnologías y las redes informáticas poseen nuevos ingredientes que convergen en la información y su uso; las redes, los equipos, los programas y los sistemas informáticos existen porque transmiten información, y el mundo actual vive alrededor de ésta y de las posibilidades que implica el acercamiento y la adquisición del conocimiento.

Las tecnologías de información y comunicación (TIC) forman parte esencial de nuestra vida cotidiana, nos proporcionan herramientas y servicios útiles en nuestro hogar, en nuestro lugar de trabajo, en nuestros quehaceres académico y educativo, casi en todos los ámbitos de nuestra vida diaria. Por supuesto, este tipo de tecnologías presentan una serie de particularidades que es preciso analizar y comprender para sacar el mejor aprovechamiento de su utilización.

En el ámbito educativo, el uso de las TIC ha generado la aparición de nuevos conceptos como la educación a distancia, la educación en línea y la teleeducación. En el contexto bibliotecario, ha hecho posible la automatización de los procesos administrativos de la biblioteca, ha permitido la aparición de las llamadas bibliotecas digitales, ha impactado en la manera de proporcionar los servicios bibliotecarios y en las colecciones de recursos informativos disponibles en una biblioteca. Aunado a ello, los usuarios de las bibliotecas y de la información presentan nuevos patrones de comportamiento generados de la utilización constante de este tipo de tecnologías en aspectos como la búsqueda y recuperación de información.

En este sentido, la presencia constante de las TIC en el entorno actual requiere que los individuos desarrollen diversas habilidades, procesos de pensamiento y actitudes, para que cualquier persona que necesite información, sea capaz de manejarla eficientemente a través del uso de estas tecnologías. En este tenor, la formación de usuarios se presenta como un nicho de oportunidad para dotar a las personas de las habilidades, conocimientos y actitudes que le permitan enfrentar sus necesidades informativas mediante el uso eficiente de las TIC. Probablemente mediante la incorporación de las aplicaciones Web 2.0 en los programas de formación de usuarios pueda alcanzarse este propósito. Sin embargo, primeramente será necesario responder a una serie de preguntas elementales:

- ¿Qué son las aplicaciones web 2.0?

- ¿Qué aspectos deben considerarse para la utilización de las aplicaciones web 2.0 en los programas de formación de usuarios?

- ¿Cuáles son las aplicaciones web 2.0 que específicamente pueden aplicarse en estos programas?

La metodología utilizada en este trabajo consiste en la utilización del análisis documental y la hermenéutica del discurso en los documentos que abordan la temática de nuestro objeto de estudio. En lo que respecta a la selección de aplicaciones web 2.0, se empleo la técnica de análisis de atributos basada en variables de uso didáctico de la aplicación.

De esta manera, el trabajo consta de cuatro apartados que abordan el estudio de la formación de usuarios; los programas de formación de usuarios; las aplicaciones Web 2.0 y su presencia en el ámbito educativo; y la utilización de las aplicaciones Web 2.0 en el diseño de programas de formación de usuarios. Finalmente se presenta un apartado de discusión y las conclusiones generadas a partir del estudio de los tópicos anteriormente mencionados.

\section{La formación de usuarios}

Para identificar el uso de las aplicaciones web 2.0 en el diseño de programas de formación de usuarios, es preciso entender cuál es la base terminológica de los componentes de la formación. En este sentido 
la formación de usuarios es el término principal del cual se desprenden los programas mencionados. Hernández Salazar (2007, p. 107), manifiesta que la formación puede entenderse desde dos perspectivas, una que se circunscribe a un proceso bibliotecario y otra que la concibe como uno didáctico.

Desde la perspectiva bibliotecaria, la formación es un proceso de transmisión lineal de conocimientos, del bibliotecario al usuario, el especialista de información "enseña" al sujeto los conocimientos necesarios para utilizar tal o cual fuente o servicio de información, sin precisar si el sujeto lo requiere o no. En este sentido, la formación impartida por el bibliotecario responde a las necesidades de la biblioteca y el programa de formación es diseñado directamente en este espacio. Desde el punto de vista didáctico, la formación pone de manifiesto la presencia de los actores que son parte del proceso enseñanza-aprendizaje de un individuo. En este sentido, el bibliotecólogo o especialista de información pretende que un usuario aprenda y no enseñarle, esta perspectiva considera las necesidades de los usuarios, a partir del aprendizaje de las diversas técnicas y estrategias para la búsqueda y recuperación de información.

Tomando en consideración estas dos perspectivas, Hernández Salazar (1998, p. 11) ha definido a la formación de usuarios como:

El proceso de intercambio de experiencias o saberes significativos sobre el uso de la información, con el fin de que la persona que la usa, de acuerdo con su proceso cognoscitivo, perciba la importancia de la información y adquiera formas de saber hacer o de resolver problemas relacionados con el acceso y uso de la información.

En el proceso de formación de usuarios intervienen una serie de factores, experiencias y saberes que son encaminados a la resolución de problemas relativos al uso y acceso a la información, estos problemas pueden manifestarse de diversa manera y su resolución dependerá estrictamente del proceso cognoscitivo de cada individuo. El proceso cognoscitivo ${ }^{1}$ del individuo le permitirá asimilar la importancia de la información y en consecuencia resolver las problemáticas que tenga en cuanto al uso y acceso a la información.

Así pues, la formación de usuarios debe entenderse como un proceso que incluye un conjunto de acciones continuas, entre las que se encuentra el intercambio de experiencias y conocimientos, encaminados a la transformación permanente del usuario a partir de la comunicación de saberes sobre el uso de la información (Rendón Giraldo N. E. y Herrera Cortés, R., 2008, p. 42).

Entonces, la formación de usuarios deberá dirigirse a una determinada comunidad de usuarios con perfiles claramente definidos que consideren las necesidades de información del usuario; aunado a ello, la formación deberá estar diseñada con la participación de bibliotecarios, profesores y otros profesionales que aporten contenidos y enriquezcan el proceso formativo del usuario.

Ahora bien, la formación de usuarios puede ser dividida en tres niveles ${ }^{2}$ :

- Primer nivel. Comunicación de conceptos relacionados con la información. En este nivel se pretende que el usuario identifique el proceso por el que pasa la información, y su importancia dentro de su formación profesional, laboral y académica.

- Segundo nivel. Orientación. Se ofrece una introducción sobre determinado servicio o unidad de información, de esta manera el usuario se familiariza con el arreglo físico de la biblioteca, sus colecciones y servicios.

- Tercer nivel. Instrucción. Permite derribar las barreas existentes entre la organización bibliotecaria y el usuario, por ejemplo: sistema de clasificación, códigos de catalogación, reglas de ordenación de catálogos, estructura de fuentes de información, entre otros. 
En función de la naturaleza del nivel de formación de usuarios se diseñaran los programas respectivos y en consecuencia se elegirán tipos determinados de tecnologías de la información y comunicación para su diseño y aplicación.

\section{Los programas de formación de usuarios}

Todo proceso de formación de usuarios requiere de una planificación didáctica ${ }^{3}$, que coordine y ordene la consecución de objetivos de aprendizaje del usuario. Los instrumentos que permiten llevar a cabo la planeación didáctica de la formación, son los denominados programas de formación de usuarios. Como su término lo indica, un programa es en general, una secuencia de instrucciones cuya ejecución resuelve una tarea concreta y produce, por tanto, un resultado (López Yepes, J., 2004, p. 342). En este sentido, la secuencia de instrucciones de un programa requiere de un método que indique el camino didáctico a seguir en la formación, y la formulación de objetivos de aprendizaje que permitan evaluar el propósito planteado, y el resultado alcanzado de la formación del usuario. Al respecto, Breivik (1982, p. 32) comenta que toda planeación de formación de usuarios deberá tener objetivos de aprendizaje claramente definidos y todo el plan deberá plasmarse en un documento que avale la ejecución de la formación.

De este modo, la planeación sistematizada de un programa de formación de usuarios incluye las siguientes etapas (Hernández Salazar, 1998. p. 14):

1. Definición del problema de formación de usuarios.

2. Determinación del entorno institucional ${ }^{4}$.

3. Determinar el perfil de necesidades de información y necesidades de formación del usuario meta (evaluación diagnóstica).

4. Establecer los objetivos del programa.

5. Elaborar los contenidos.

6. Seleccionar las técnicas y medios didácticos

7. Elaborar los medios didácticos.

8. Implementar el programa.

9. Evaluar el programa (evaluación formativa y sumativa).

Evidentemente, todo proceso de planeación debe considerar factores como la comunidad, personal, presupuesto, apoyo y facilidades prestadas de la entidad a la que será dirigido el programa. Asimismo, para el desarrollo de algunas de las etapas del diseño del programa de formación de usuarios van a requerir de una serie de tecnologías. En este sentido, diversos tipos de aplicaciones web pueden ser utilizadas y aplicadas para llevar a cabo el diseño de dichos programas. Sin embargo, antes de utilizarlas y aplicarlas es necesario conocerlas, analizarlas y evaluar sus alcances y limitaciones en lo que a la utilidad que pueden tener en el díselo de programas de formación de usuarios.

\section{Aplicaciones Web 2.0 y educación}

Antes de adentrarnos en el tema de las aplicaciones Web 2.0 y su utilización en el entorno educativo, es necesario analizar la definición de tecnologías de la información y comunicación mediante el estudio de 
sus componentes terminológicos, ya que evidentemente, las aplicaciones Web forman parte del gran espectro de tecnologías de información y comunicación.

En este sentido, el término tecnologías de la información y comunicación está integrado por tres conceptos:

- La tecnología. Se entiende como el producto de la aplicación de la ciencia al desarrollo de artefactos, máquinas y procedimientos que pueden llegar a mejorar algunos aspectos de la vida del ser humano.

- La información. Se refiere al conjunto de datos (en sus distintos formatos y presentados en un contexto) que se transmiten entre los individuos, quienes los interpretan. Es decir, la información solo existe cuando los datos adquieren significado.

- La comunicación. Es un proceso a través del cual los individuos intercambian información y se influencian unos a los otros a partir de un sistema común de símbolos y signos.

Tomando en consideración estos conceptos podemos definir a las tecnologías de información y comunicación como:

Herramientas, procesos y productos del conocimiento humano que pueden mejorar la información y comunicación, bajo la condición de que, en contextos específicos, su uso fortalezca y contribuya a desarrollar procesos cognitivos; es decir, que coadyuve a que las personas se relacionen, colaboren y aprovechen su capacidad de reflexionar lógica y creativamente teniendo el aprendizaje como propósito fundamental (Zubieta, Bautista y Quijano, 2009. p. 19).

Las TIC tienen un espectro muy amplio de aplicación, actualmente la mayoría de los contextos del desarrollo humano, requieren del uso de estas tecnologías, ya sea como herramienta, proceso o producto que les permitan llevar a cabo las actividades que están inmersas en su quehacer cotidiano. En función del contexto donde las TIC sean aplicadas, será necesario considerar sus atributos.

Por lo tanto, será necesario tomar en cuenta una clasificación de tecnologías de la información y comunicación contemplando su naturaleza informativa y comunicativa, esta clasificación puede resumirse en términos generales de la siguiente manera:

\begin{tabular}{|l|l|}
\hline \multicolumn{1}{|c|}{ Tecnologías de información } & \multicolumn{1}{c|}{ Tecnologías de comunicación } \\
\hline Computadoras & Teléfono \\
\hline Software & Teléfonos inteligentes \\
\hline Hardware & Internet \\
\hline Soportes de almacenamiento de información & Televisión \\
\hline Aplicaciones Web & Radio \\
\hline
\end{tabular}

Figura 1: Clasificación de tecnologías de información y comunicación.

Fuente: El autor (2013)

Como puede observarse, nuestra clasificación de TIC maneja una división entre tecnologías de la información y tecnologías de la comunicación. Ya que en conjunto estas dos tecnologías forman parte del amplio grupo de las tecnologías de información y comunicación. En este sentido, es necesario considerar que las tecnologías de la información se refieren a un conjunto de herramientas utilizadas 
para crear, almacenar, intercambiar, recuperar y visualizar la información en todas sus formas, así como los conocimientos necesarios para crearlas, mejorarlas, adaptarlas y modificarlas. A su vez, las tecnologías de la comunicación se refieren al conjunto de herramientas creadas para la transmisión y recepción de datos, ideas, información y mensajes.

Las aplicaciones Web, forman parte de las tecnologías de la información y comunicación. Una aplicación Web en términos concretos se define como un programa o conjunto de programas informáticos que ayudan a los usuarios de una computadora a procesar una tarea específica. Una aplicación Web está compuesta por una interfaz de usuario y es accesible normalmente a través de un navegador Web. Ahora bien, el termino aplicación Web 2.0 ha nacido a la par de la evolución de la propia Web. La web es un medio relativamente joven y en constante evolución.

Como manifiestan Castellanos et al. (2011):

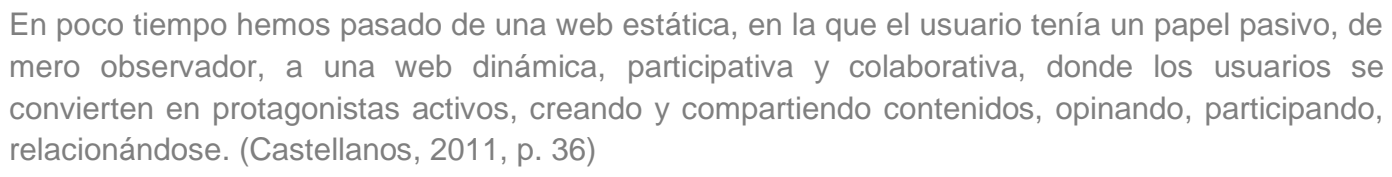

A esta nueva Web se le denomina Web 2.0 o Web social y ofrece grandes alcances en el ámbito formativo y educativo. La Web 2.0 se ha convertido en el escenario en el que convergen cuatro elementos fundamentales: los usuarios, los servicios, las aplicaciones y los medios. Por lo tanto, tomando en consideración los argumentos anteriores, podemos entender a las aplicaciones Web 2.0 como un programa o conjunto de programas informáticos, que están compuestos de una interfaz de usuario, y son accesibles mediante la intervención de un navegador web que permite al usuarios crear, publicar y compartir contenidos ya sea de manera individual o colectiva.

Es importante considerar que ese tipo de aplicaciones permiten la participación y colaboración de los usuarios, a través de la construcción de relaciones.

Ahora bien, entre los alcances y las limitaciones de las aplicaciones Web 2.0 podemos encontrar los siguientes argumentos:

Alcances

- Amplia disponibilidad. Están disponibles casi desde cualquier lugar con conexión a internet.

- Son multiplataforma. Es decir, son funcionales independientemente del sistema operativo que se utilice, e incluso pueden utilizarse desde cualquier dispositivo, ya sea este una computadora o bien, un teléfono inteligente.

- Actualización periódica. Son aplicaciones que cuentan con un servicio de actualización que funciona de manera periódica y automática.

- Menor requerimiento de hardware y software. Para el uso de la aplicación solo se requiere el uso de un dispositivo y un navegador Web.

- Colaboración. En el entorno de trabajo de la aplicación pueden participar varios usuarios simultáneamente y de manera remota. 


\section{Limitaciones}

- La información creada por la aplicación puede ser consultada por terceros. En ocasiones la información que se es creada con la aplicación puede ser consultada y utilizada por personas ajenas al grupo colaborativo.

- Cambios de condiciones en el servicio de la aplicación. Puede que las clausulas del servicio de I aplicación cambien de manera inesperada, y pasen de ser una aplicación gratuita a una comercial.

- Copias de seguridad. Es necesario mantenerse alertas ante eminentes pérdidas de información en el servicio de la aplicación, lo que hace necesario crear copias de seguridad con mayor frecuencia.

Considerar los alcances y limitaciones que tienen las aplicaciones web 2.0 nos permitirá llevar a cabo una mejor evaluación de su utilización en entornos específicos. Sin embargo es preciso entender que los alcances y limitaciones dependerán en gran medida de la propia naturaleza de la aplicación. Ahora bien, entre las implicaciones educativas y formativas de las aplicaciones Web 2.0, podemos resaltar aquellas que nos permiten observar la transformación de los entornos de enseñanza aprendizaje de los individuos. Al respecto la ONU (2013) manifiesta que "las tecnologías de la información y la comunicación pueden contribuir al acceso universal a la educación, la igualdad en la instrucción, el ejercicio de la enseñanza y el aprendizaje de calidad y el desarrollo profesional de los docentes, así como a la gestión dirección y administración más eficientes del sistema educativo".

La aplicación de las TIC en los entornos educativos ha propiciado la aparición de término como la educación a distancia, la teleformación y un número bastante amplio de conceptos que intentan explicar la integración de estas tecnologías en los diversos entornos educativos de la sociedad.

Las aplicaciones Web 2.0 pueden propiciar la construcción de espacios de enseñanza aprendizaje ricos en fuentes de información (a través de la red social donde el conocimiento no está cerrado), a su vez suponen una alternativa a la jerarquización y unidirecionalidad tradicional de los entornos formativos. Pero también, su utilización implica nuevos roles para docentes y alumnos orientados al trabajo autónomo y colaborativo, crítico y creativo, la expresión personal, la investigación y compartir recursos, crear conocimiento y adquirir un aprendizaje significativo. Estas aplicaciones pueden proporcionar espacios en línea para el almacenamiento, clasificación y publicación/difusión de contenidos textuales y audiovisuales, a los que luego todos podrán acceder. Además, su correcta utilización facilitará la realización de nuevas actividades de aprendizaje y de evaluación y la creación de redes de aprendizaje.

\section{Uso de aplicaciones Web 2.0 en los programas de formación de usuarios}

La filosofía de la Web 2.0 se basa en el componente social y potencia la comunicación, la colaboración y el intercambio. En este sentido si la docencia y el proceso de enseñanza y aprendizaje se basan en la comunicación, sería absurdo no aprovechar la potencia y el alcance de la Web para desarrollar valores de participación, colaboración y construcción colectiva del conocimiento.

Podemos utilizar las aplicaciones Web 2.0 en los programas de formación de usuarios de diversas maneras. Una de ellas es haciendo uso de las aplicaciones que tenemos a disposición en el gran entorno de la web. A continuación presentamos algunas aplicaciones Web 2.0 que pueden utilizarse en el desarrollo de programas de formación de usuarios de la información: 
Tabla 2. Aplicaciones Web 2.0 para su aplicación en los programas de usuarios de la información.

\begin{tabular}{|c|c|}
\hline Nombre la aplicación & Descripción \\
\hline Prezi & $\begin{array}{l}\text { Es una aplicación multimedia para la creación de presentaciones similar a Power } \\
\text { Point de Microsoft. Ofrece la opción de crear presentaciones de manera colectiva, } \\
\text { dinámicas y compartirlas con tus contactos a través de la Web. Disponible en: } \\
\text { http://prezi.com/ }\end{array}$ \\
\hline Blogger & $\begin{array}{l}\text { Es una aplicación que permite crear y publicar una bitácora en línea. Permite integrar } \\
\text { los servicios de Google en la estructura de la bitácora creada. Disponible en: } \\
\text { www.blogger.com/ }\end{array}$ \\
\hline Wikispaces & $\begin{array}{l}\text { Aplicación gratuita que permite la construcción de wikis. Las wikis5 creadas con esta } \\
\text { aplicación pueden ser de acceso libre o restringido. Permite la creación colectiva de } \\
\text { contenidos y ofrece diversas herramientas didácticas. Disponible en: } \\
\text { www.wikispaces.com }\end{array}$ \\
\hline Piktochart & $\begin{array}{l}\text { Aplicación que permite la creación de infografías } 6 \text { ofrece al usuario un catálogo de } \\
\text { plantillas predefinidas a las que puede añadir contenido y modificar la estructura con } \\
\text { flexibilidad. Además, añade un catálogo de formas e imágenes clasificadas por áreas } \\
\text { temáticas para personalizar las infografías. Disponible en: www.piktochart.com }\end{array}$ \\
\hline Mindomo & $\begin{array}{l}\text { Aplicación para la creación de mapas mentales. Permite crear, editar y compartir } \\
\text { mapas mentales a través de la Web. Ofrece la posibilidad de trabajar colectivamente } \\
\text { y de manera simultánea sobre un mapa en específico. Disponible en: } \\
\text { www.mindomo.com }\end{array}$ \\
\hline Edmodo & $\begin{array}{l}\text { Aplicación social y educativa gratuita, que permite la comunicación entre los alumnos } \\
\text { y profesores en un entorno cerrado y privado a modo de microblogging. Permite la } \\
\text { construcción de aulas virtuales de manera sencilla y dinámica, ofrece una amplia } \\
\text { gama de herramientas didácticas. Está disponible para iOS y Android, además de su } \\
\text { clásica versión en línea. Disponible en: www.edmodo.com }\end{array}$ \\
\hline Coursera & $\begin{array}{l}\text { Aplicación de educación virtual desarrollada por académicos de la Universidad de } \\
\text { Stanford con el fin de brindar oferta de educación masiva a la población mundial. } \\
\text { Esta aplicación permite la creación de cursos a través de Internet. Disponible en: } \\
\text { https://www.coursera.org }\end{array}$ \\
\hline Youtube & $\begin{array}{l}\text { Aplicación que permite subir, reproducir y compartir videos a través de su sitio web } \\
\text { oficial. Es una de las aplicaciones más populares en la web } 2.0 \text { y su potencial puede } \\
\text { ser aplicado a las actividades didácticas de enseñanza aprendizaje. Disponible en: } \\
\text { www.youtube.com }\end{array}$ \\
\hline Diigo & $\begin{array}{l}\text { Aplicación que funciona como un sistema personalizado de gestión de información. } \\
\text { Ofrece a sus usuarios la posibilidad de utilizar y compartir marcadores web, bloc de } \\
\text { notas post-it, archivos de imágenes y documentos, así como selección de textos } \\
\text { destacados en entornos Web. Disponible en: https://www.diigo.com }\end{array}$ \\
\hline Screenr & $\begin{array}{l}\text { Aplicación que permite realizar grabaciones de pantalla de la computadora de forma } \\
\text { online, podemos grabar todo lo que vamos haciendo en la computadora a la vez que } \\
\text { explicamos el proceso de construcción de aprendizajes. Por lo tanto, es ideal para } \\
\text { elaborar videotutoriales sobre cualquier contenido que queramos explicar a los } \\
\text { alumnos. Disponible en: www.screenr.com }\end{array}$ \\
\hline
\end{tabular}

Fuente: El autor ( 2013) 
Ahora bien, ¿Cómo pueden utilizarse estas aplicaciones en los programas de formación de usuarios de la información? Las aplicaciones que hemos reunido en este trabajo son solo una pequeña parte del gran universo de los recursos que existen en la Web. Las aplicaciones pueden utilizarse para la creación de medios didácticos, o bien como elementos integrantes de la planeación didáctica de los programas. Su nivel de utilización y aplicación en los programas, dependerá en gran medida del dominio que se tenga en la manipulación de la aplicación. Por este motivo, las aplicaciones Web 2.0 son una herramienta para explotar la creatividad del formador en contexto enseñanza aprendizaje del individuo que será formado. Las aplicaciones Web 2.0 establecen una sinergia y convergencia a través de las innovaciones en las dimensiones tecnológicas, organizacionales y pedagógicas, dan soporte a aspectos como:

- El aprendizaje y la enseñanza

- Los logros individuals

- Las redes

- La diversidad

- Y a la sociedad para potenciar las habilidades del alumno

Asimismo, el surgimiento de estas nuevas tecnologías, ha afectado distintos aspectos del comportamiento del individuo, algunos aspectos han sido positivos y otros tantos, negativos. Sin embargo, los propios individuos han sido los protagonistas del cambio, un cambio fundamentado en las necesidades de las personas, que ha dado pauta a la creación de espacios de integración entre lo social y lo tecnológico, donde las nuevas aplicaciones proporcionan servicios y auxilian en el desarrollo de tareas a los individuos. En este sentido, el uso de las aplicaciones Web 2.0 en los programas de formación de usuarios de la información puede tener varias aristas:

- Las aplicaciones pueden fungir como herramienta para construcción de los contenidos de los programas

- Pueden utilizarse como objetivo de aprendizaje, es decir. Construir programas de formación de usuarios basados en la enseñanza de este tipo de tecnologías

- Eventualmente pueden aplicarse para cambiar los paradigmas de la formación, es decir poniendo en marcha programas de formación a distancia.

El uso de TIC en los procesos de enseñanza-aprendizaje de los individuos es fundamental para el trabajo diario de los individuos. Sin embargo, es necesario que docentes y alumnos se adapten a las exigencias que demanda la utilización de estas tecnologías.

\section{Discusión y conclusiones}

El uso de las aplicaciones web 2.0 en los programas de formación de usuarios de la información requiere que los actores del proceso de enseñanza-aprendizaje desarrollen una serie de competencias que les permitan explotar todos los alcances de estas tecnologías. 
Trabajando con las aplicaciones de la Web 2.0, los estudiantes y formadores serán más autónomos en el acceso a la información y la construcción de sus conocimientos. No obstante necesitarán desarrollar unas competencias específicas:

Competencias digitales: que le permitan navegar (buscar, seleccionar, y evaluar la información disponible en la Web), procesar la información con los medios informáticos para elaborar su conocimiento, expresarse y comunicarse con otros en el entorno digital, conocer los riesgos de la web (plagio, anonimato).

Competencias sociales: que le permitan trabajar en equipo, respetar todas las manifestaciones del conocimiento, tener responsabilidad en el desarrollo de nuevos conocimientos.

Competencias comunicacionales: que le permitan privilegiar y valorar la correcta utilización del lenguaje, fomentar el trabajo colaborativo.

Competencias didácticas: aplicar modelos didácticos de uso de las aplicaciones Web 2.0, bien contextualizados a los alumnos y claros objetivos de aprendizaje que persigan la formación integral del estudiante.

Los retos del siglo XXI, pueden enfrentarse con educación de calidad. Con una formación, que además de los aprendizajes tradicionales, enseñe a los individuos a utilizar, valorar y calificar la información que se encuentra en su entorno. Sin duda, actualmente los fenómeno derivados del uso y aprovechamiento de la información son más constantes.

Las tendencias nos permiten apreciar que la tecnología y la información son la integración perfecta para alcanzar el desarrollo. Por este motivo, los individuos requieren, más que en otras épocas, una formación integral que les permita enfrentar sus problemas relacionados con el uso de la información y de la tecnología para su tratamiento. Las tecnologías de la web 2.0 y su integración en los programas de formación de usuarios de la información, representan un nicho de oportunidad para los bibliotecólogos. Una oportunidad para alcanzar el futuro y romper viejos paradigmas de la formación de usurios.

\section{Bibliografia}

Breivik, P. (1982). Planning the library instruction program. Chicago: ALA.

Castellanos, J. et Al. (2011). Las TIC e n la educación. Madrid: Anaya.

Hernández Salazar, P. (1998). La formación de usuarios de información en instituciones de educación superior. México: UNAM, CUIB.

Hernández Salazar, P. (2007). La relación entre los estudios y la formación de usuarios de la información. Revista general de información y documentación, 17, (2). p. 103-121.

López Yepes, J. (2004). Diccionario enciclopédico de ciencias de la documentación. Madrid, Síntesis.

Organización de las Naciones Unidas. (2013). Las TIC en la educación. Recuperado de

http://www.unesco.org/new/es/unesco/themes/icts/

Perrone, G. y Propper, F. (2007). Diccionario de educación. Argentina: Alfagrama.

Rendón Giraldo N. E. y Herrera Cortés, R. (2008). Hacia una formación de usuarios de la información en entornos locales. Información, cultura y sociedad, 19. p. 35-62.

Zubieta García, J., Bautista Godínez, T. y Quijano Solís, A. (2012). Aceptación de las TIC en la docencia: una tipología de los académicos de la UNAM. México: UNAM, Porrúa. 


\section{Notas}

${ }^{1}$ El proceso cognoscitivo es un recurso de la conciencia en el que mediante fenómenos biológicos y mecanismos psicológicos generan la obtención de un conocimiento elaborando de esta manera el contenido racional de la personalidad.

2 Estos niveles han sido propuestos por Hernández Salazar y se relacionan con el proceso cognoscitivo del aprendizaje significativo del usuario.

3 Perrone y Propper, 2007. p. 308. Definen a la planificación didáctica como un proceso que permite organizar el trabajo docente mediante la anticipación de aquello que se va a realizar en el aula. Se trata de la previsión del camino a seguir y los medios con que se cuenta para llegar a los objetivos propuestos. En primer lugar, se establecen las intenciones educativas (objetivos y propósitos), además se especifican las actividades. También debe contemplarse el cómo, es decir con qué estrategias de enseñanza y a través de que técnicas los alumnos se apropiarán de los conocimientos. Resulta un elemento clave para alcanzar un aprendizaje significativo y puede ayudar al docente a alcanzar con eficiencia los objetivos.

4 Mediante la obtención de datos como objetivos general y específicos, estructura organizacional, políticas, programas, recursos (humanos, económicos, físicos) y procesos (tanto de la institución en la que está inserta la unidad de información como de la unidad misma). También se debe considerar el entorno físico: instalaciones y tamaño.

5 El término wiki proviene del hawaiano que significa rápido. Es un sitio que se edifica de manera colectiva y horizontal, desde el navegador web y en tiempo real. Los autores del wiki pueden editar, publicar, modificar y construir el conocimiento. Las Wikis en el entorno de la Web 2.0 son de las aplicaciones más populares. Su ejemplo más representativo es Wikipedia, pero es sólo un ejemplo más de los muchos que se encuentran en la Web.

${ }^{6}$ López Yepes, manifiesta que una infografía es la aplicación de la informática a la documentación gráfica, tras el tratamiento de la imagen. También puede concebirse como la técnica que permite la creación de imágenes 0 gráficos por computadora. Véase: López Yepes, J. (2004). Diccionario de Documentación. Madrid: Síntesis. p. 58. Desde nuestro punto de vista fundamentado en los objetivos de aprendizaje de este programa, una infografía es un recurso de información que utiliza texto, imágenes y gráficos que se relacionan entre sí para explicar cualquier tópico o tema de interés.

\section{Datos del autor}

\section{Eder Ávila Barrientos}

Asistente de Coordinación del Posgrado en Bibliotecología y Estudios de la Información de la Universidad Nacional Autonoma de México. Maestro en Bibliotecologia y Estudios de la Información por la UNAM. Profesor de la asignatura introducción a la investigación. Universidad Nacional Autónoma de México. Facultad de Filosofía y Letras. Colegio de Bibliotecología.

eavila86@gmail.com

Recibido - Received: 2013-12-11

Aceptado - Accepted: 2014-06-30

\section{$(\mathrm{cc}) \mathrm{EY}$}

This work is licensed under a Creative Commons Attribution 4.0

United States License.

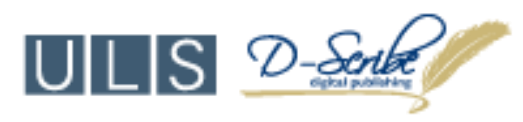

This journal is published by the University Library System of the University of Pittsburgh as part of its D-Scribe Digital Publishing Program and is cosponsored by the University of Pittsburgh Press. 